\title{
Arctiini Leach, [1815] (Lepidoptera, Erebidae, Arctiinae) of the Brazilian Amazon. IV - Subtribe Euchromiina Butler, 1876
}

\author{
José Augusto Teston ${ }^{1 * \text { (D) \& Viviane Gianluppi Ferro }}{ }^{2}$ \\ ${ }^{1}$ Universidade Federal do Oeste do Pará, Instituto de Ciências da Educação, Laboratório de Estudos de \\ Lepidópteros Neotropicais, Rua Vera Paz s/n, CEP 68040-255, Santarém, PA, Brasil \\ ${ }^{2}$ Universidade Federal de Goiás, Instituto de Ciências Biológicas, Departamento de Ecologia, Caixa Postal \\ 131, CEP 74001-970, Goiânia, GO, Brasil \\ *Corresponding author: José Augusto Teston, e-mail: jateston@gmail.com
}

TESTON, J. A., FERRO, V. G. Arctiini Leach, [1815] (Lepidoptera, Erebidae, Arctiinae) of the Brazilian Amazon. IV - Subtribe Euchromiina Butler, 1876. Biota Neotropica. 19(4): e20190747. http://dx.doi.org/10.1590/1676-0611-BN-2019-0747

\begin{abstract}
The Euchromiina moths comprise 735 species in the neotropics. Here we provide a list of Euchromiina species from the Brazilian Amazon. The list was produced from specimens deposited in the most important Brazilian collections and from literature data. Nearly 30\% (219) of the neotropics Euchromiina species were recorded, including 13 new occurrences for the Brazilian Amazon. Santarém and Belém were the municipalities with the highest number of species records, with 96 and 74, respectively. Although the number of Euchromiina records is high in the Amazon, this value is underestimated because the fauna was never sampled in the vast majority of the biome. This is a worrying scenario because the Amazon has the world highest absolute rate of forest reduction. In order to suggest efficient conservation policies for the Amazon Arctiinae fauna, it is urgent to intensify the sampling effort in this biome.
\end{abstract}

Keywords: Amazon, Tiger moths, Inventory, Noctuoidea, Wasp Moths.

\section{Arctiini Leach, [1815] (Lepidoptera, Erebidae, Arctiinae) da Amazônia Brasileira. IV - Subtribo Euchromiina Butler, 1876}

Resumo: As mariposas Euchromiina compreendem 735 espécies na região Neotropical. Neste trabalho, nós apresentamos uma lista das espécies de Euchromiina que ocorrem na Amazônia brasileira. A lista foi produzida através de observação de espécimes depositados nas mais importantes coleções brasileiras e também através de dados da literatura. Aproximadamente 30\% (219) das espécies de Euchromiina que ocorrem na região Neotropical foram registradas, incluindo 13 novas ocorrências para a Amazônia brasileira. Santarém e Belém foram os municípios com maior número de espécies registradas, com 96 e 74, respectivamente. Embora o número de registros de Euchromiina seja considerado alto na Amazônia, este valor é subestimado visto que a fauna nunca foi amostrada na imensa maioria do bioma. Este cenário é preocupante, pois a Amazônia possui a maior taxa absoluta de redução florestal do mundo. A fim de podermos sugerir políticas de conservação eficientes para a fauna de Arctiinae da Amazônia, é urgente que se intensifique o esforço de coleta neste bioma.

Palavras-chave: Amazônia, mariposas-tigre, Inventário, Noctuoidea, mariposas-vespa. 


\section{Introduction}

Tropical rainforests support some of the hightest species richness of the Earth and correspond to $60 \%$ of the hotspots defined by Myers et al. (2000). The Amazon represents $40 \%$ of the tropical rainforest areas (Aragão et al. 2014). It covers part of nine countries in South America, with $69 \%$ of this area in Brazil (Vieira et al. 2008). However, the original area of the Brazilian Amazon has been reduced by 20\% (INPE 2015).

Lepidoptera inventories in the Brazilian Amazon are scarce (Santos et al. 2008). Although there have been scientific publications on Amazonian Lepidoptera since the 19th century, many earlier inventories in the Brazilian Amazon do not provide an accurate description of the collection sites, which significantly restricts the use of their data (Casagrande et al. 2012). Several recent inventories also have problems, such as being made in a short period of time and/or poorly distributed in space (usually along major rivers or near urban centers).

Arctiinae was worldwide Lepidoptera taxa (Heppner 1991). With approximately 11,000 species, the tiger moths are divided in four tribes (Zahiri et al. 2012), but only Arctiini and Lithosiini occurred in the Neotropics. Arctiini are distributed in seven subtribes: Arctiina, Callimorphina, Spilosomina, Phaegopterina, Pericopina, Ctenuchina and Euchromiina (Weller et al. 2009, Vincent \& Laguerre 2014).

The Euchromiina moths are found mainly in the neotropics and comprises approximately 68 genera and 735 species (Weller et al. 2009). Several species have nocturnal activity, but some species are exclusively diurnal (Hagmann 1938). Many species of Euchromiina form mimetic rings with butterflies, beetles, and especially wasps (Simmons 2009). Their wings can have areas or be totally transparent, closely resembling the wings of Hymenoptera. Moreover, some species simulate the petiole of Hymenoptera by reducing of abdomen sclerites and increasing thorax sclerites (Simmons 2009). Males of several species have modified scales below the second abdominal segment that can be released in dangerous situations or during mating (Yack 2004). Some Euchromiina use ultrasound in courtship instead of or in combination with feromonal cues (Sanderford et al. 1998). Several Euchromiina larvae are brilliantly coloured (Weller et al. 2009) and feed on several plant families. Adults of some species are pharmacophagous on pyrrolizidine alkaloids (Conner \& Jordan 2009).

We present a list of Euchromina moths occurring in the Brazilian Amazon. We make the species list mainly from specimens deposited in the most important Brazilian collections and also from literature data. This study is a continuation of Teston \& Ferro (2016a, b) and Teston et al. (2019) and aims to increase knowledge of the diversity of Arctiinae in the Amazon region.

\section{Materials and Methods}

We intensively searched the literature and examined specimens from entomological collections of the Instituto Nacional de Pesquisas na Amazônia (INPA; Manaus), Museu Paraense Emilio Goeldi (MPEG; Belém), Coleção Becker (VOB; Camacan), Coleção Entomológica Padre Jesus Santiago Moure of the Universidade Federal do Paraná (DZUP; Curitiba), Fundação Instituto Oswaldo Cruz (FIOC; Rio de Janeiro), Museu de Zoologia of the Universidade de São Paulo (MZUSP; São Paulo), Museu Nacional of the Universidade Federal do Rio de Janeiro (MNRJ; Rio de Janeiro), and Laboratório de Estudos de Lepidópteros Neotropicais (LELN) of the Universidade Federal do Oeste do Pará (UFOPA; Santarém). To identify the species, we used literature (Hampson 1898, 1914, Seitz 1919-1925) and specimens deposited in the visited collections. The systematic organization to generic level follows Hampson (1898) and Weller et al. (2000), with updates of the generic names according Watson et al. (1995) and corrections of Pinheiro \& Duarte (2013), Pinheiro \& Gaal-Haszler (2015) and Pinheiro (2016).

The geographical coordinates of the localities in the Brazilian Amazon with Arctiinae records were obtained from the Geo Loc tool of "Species Link date \& tools" (http://splink.cria.org.br/geoloc) and Google Earth (https://earth.google.com/web/). The list is organized alphabetically. Species and records without precise location data, and those from locations that belong to more than one biome (e.g., Cerrado and Amazon) were not included in the list.

\section{Results}

Our research generated a list of 219 Euchromiina species (Table 1), including 13 new occurrences for the Brazilian Amazon (indicated by "NEW"). Eighteen species appear as new records for the municipalities and their respective States (indicated by "AMZ”). In total, 71 (9.2\%) Amazonian municipalities had Euchromiina species records (Table 2 and Figure 1). Santarém (PA), Belém (PA), and São Félix do Xingu (PA) were the municipalities with the highest number of species, with 96,74 , and 47 , respectively.

We recorded 49 genera, of which 19 were monospecific. The genera Cosmosoma Hübner, [1823] presented the highest number of species (27), followed by Saurita Herrich-Schäffer, [1855] (19) and Leucotmemis Butler, 1876 (12). The species with the highest number of locality records were Belemnia eryx (Fabricius, 1775) and Isanthrene porphyria (Walker, 1854) (with 15), followed by Orcynia calcarata (Walker, 1854) (14), Cosmosoma telephus (Walker, 1854) and Histioea proserpina (Hübner, 1827) (13). Ninety-one species (41.4\%) occurred in only one locality (Table 1).

\section{Discussion}

The number of Euchromiina species recorded for the Brazilian Amazon was high. It corresponds to $29.8 \%$ of neotropics Euchromiina fauna (735, Weller et al. 2009) and was more than two times higher than the Mexican (112, Hernández-Baz et al. 2013), the Brazilian Cerrado (76, Ferro et al. 2010), and Rio Grande do Sul Brazilian State (60, Ferro $\&$ Teston 2009) Euchromiina richness.

The explanation of Santarém and Belém are the Amazon municipalities with the highest number of Euchromiina records probably are related to the proximity and access to the collection sites, which allow a larger sample effort. Belém is the capital of the state of Pará and has an important museum (Museu Paraense Emílio Goeldi, founded in 1866) and other research institutions, as well as easy access to sampling sites. Santarém was widely sampled by H. Zerny and G. Hagmann (Zerny 1931, Hagmann 1938). Hagmann resided in Santarém and Zerny spent a season collecting moths there. Belém was also the second municipality with the highest number of Phaegopterina, Pericopina and Ctenuchina records (Teston \& Ferro 2016a, b, Teston et al. 2019). 
Table 1. Euchromiina (Erebidae, Arctiinae, Arctiini) species of the Brazilian Amazon. The record column shows the Brazilian state in abbreviated form followed by municipality. The name of the locality is enclosed in braces and the author of the first record is in parentheses. $*$ New record. States abbreviations: AC $=$ Acre, $\mathrm{AM}=$ Amazonas, $\mathrm{AP}=$ Amapá, $\mathrm{MA}=$ Maranhão, $\mathrm{MT}=$ Mato Grosso, $\mathrm{PA}=$ Pará, $\mathrm{RO}=$ Rondônia and $\mathrm{RR}=$ Roraima.

\begin{tabular}{cl}
\hline \multicolumn{2}{l}{ Species } \\
\hline 1. & Abnormipterus abnormis (Hampson, 1898) \\
3. & Autochloris bijuncta (Walker, 1856) \\
4. & Autochloris caunus (Cramer, [1779]) \\
5. & Autochloris completa (Walker, 1854) \\
6. & Autochloris crinopoda Kaye, 1918 NEW \\
7. & Autochloris ectomelaena Hampson, 1898 \\
8. & Autochloris enagrus (Cramer, [1779]) \\
9. & Autochloris proterva (Draudt, 1916) \\
10. & Autochloris simplex (Walker, 1856) \\
11. Autochloris solimões Schaus, 1924 \\
12. Belemnia eryx (Fabricius, 1775)
\end{tabular}

13. Belemnia inaurata (Sulzer, 1776) ${ }^{\mathrm{AMZ}}$

14. Belemnia ochriplaga Hampson, 1901

15. Belemniastis eucyane (R. Felder, 1875)

16. Belemniastis whiteleyi (Druce, 1888) NEW

17. Bodosa tina (Walker, 1854)

18. Calonotos acutipennis Zerny, 1931

19. Calonotos aequimaculatus Zerny, 1931

20. Calonotos angustipennis Zerny, 1931

21. Calonotos chalcipleura Hampson, 1898

22. Calonotos helymus (Cramer, [1775])

23. Calonotos hoffmannsi (Rothschild, 1911)

24. Calonotos longipennis Rothschild, 1911

25. Calonotos phlegmon (Cramer, [1775])

\section{Record}

AM, [Boa Vista do Ramos] \{Massauari\} (Hampson 1898)

AM, Tefé \{Ega\} (Walker [1865]); PA, [Belém] (Walker 1856)

AM, São Paulo [de Olivença] (Zerny 1931); PA, Santarém \{Taperinha\} (Zerny 1931)

AM, Benjamin Constant*, Tefé \{Ega\} (Walker [1865]); MA, Açailândia*; RO, Cacaulândia*

AM, [Eirunepé] \{Juruá river, São Felipe\} (Zerny 1931), São Paulo de Olivença*; PA, [Belém] (Walker 1854a)

AM, Benjamin Constant*, Maués*; PA, Novo Progresso $\{\text { Cachimbo }\}^{*}$,

AM, Itamar[a]t[i] (Rothschild 1931), upper Amazonas [river] (Hagmann 1938); PA, Santarém \{Taperinha\} (Hagmann 1938)

AM, Itacoatiara*, Tefé \{Ega\} (Hampson 1898); PA, Óbidos*, Santarém*, São Félix do Xingu \{Serra do Pardo National Park\} (Teston \& Correa 2015)

PA, Belterra \{National Forest of Tapajós\} (Freitas 2014)

AM, Tefé (Zerny 1931); PA, [Belém] (Walker 1856), Santarém \{Mojú\} (Zerny 1931)

AM, [Tefé] \{Mouth of Rio Tefé and Rio Solimões\} (Schaus 1924)

AM, Borba*, Fonte Boa (Rothschild 1910), Humaitá (Rothschild 1910), São Paulo de Olivença*, Tefé (Rothschild 1910); MA, Açailândia*; MT, Sinop*; PA, Itaituba*, Parauapebas \{Serra Norte, Carajás\}*, Prainha (Butler 1878), Santarém*, Tapajós [river] (Hampson 1901); RO, Ariquemes*, Cacaulândia*, Pimenta Bueno*, Porto Velho \{Aliança\} (Rothschild 1910)

AM, Manicoré*, São Gabriel da Cachoeira*, Tefé*; AP, Serra do Navio*; MT, Sinop*; PA, Belém*, Santarém*

AM, Fonte Boa (Rothschild 1910), Humaitá (Rothschild 1910), Manaus*, Tefé (Rothschild 1910); PA, [Belém] (Hampson 1901), Belterra \{National Forest of Tapajós\} (Freitas 2014), Ju[r]ut[i] (Rothschild 1910), Prainha (Hampson 1901), Santarém*; RO, Porto Velho \{Aliança\} (Rothschild 1910)

RO, Porto Velho \{Aliança\} (Hampson 1920)

AM, Benjamin Constant*

AM, Maués (Machado Filho \& Rêgo Barros 1969), Tefé \{Ega\} (Machado Filho \& Rêgo Barros 1969); PA, Óbidos (Machado Filho \& Rêgo Barros 1969), Santarém \{Taperinha\} (Machado Filho \& Rêgo Barros 1969)

PA, Belterra \{National Forest of Tapajós\} (Freitas 2014), Santarém \{Taperinha\} (Zerny 1931)

PA, Altamira \{Serra do Pardo National Park\} (Teston \& Correa 2015), Belterra \{National Forest of Tapajós\} (Freitas 2014), Santarém \{Taperinha\} (Zerny 1931), São Félix do Xingu \{Serra do Pardo National Park\} (Teston \& Correa 2015)

PA, Belterra \{National Forest of Tapajós\} (Freitas 2014), Santarém \{Taperinha\} (Zerny 1931), São Félix do Xingu \{Serra do Pardo National Park\} (Teston \& Correa 2015)

AM, Barcelos \{Moura\}*, Manaus*; AP, Serra do Navio*; MA, Açailândia*; MT, Aripuanã*; PA, Belém*, Novo Progresso $\{\text { Cachimbo }\}^{*}$, Parauapebas $\{$ Serra Norte, Carajás $\}$ *, Óbidos*, Santarém \{Taperinha\} (Zerny 1931); RO, Cacaulândia*

AP, Serra do Navio*; PA, Santarém (Valente et al. 2018)

PA, Itaituba to Óbidos (Rothschild 1911), Itaituba (Hampson 1914)

PA, São Félix do Xingu \{Serra do Pardo National Park\} (Teston \& Correa 2015)

AM, Manaus*, São Paulo [de Olivença] (Zerny 1931); AP, Serra do Navio*; MA, Açailândia*; MT, Aripuanã*; PA, [Belém] (Hampson 1898), Capitão Poço*, Marabá*, Novo Progresso $\{\text { Cachimbo }\}^{*}$, Parauapebas $\left\{\right.$ Serra Norte, Carajás ${ }^{*}$, Óbidos*; RO, Cacaulândia*, Jarú* 
Teston \& Ferro

Continuation Table 1.

\begin{tabular}{ll}
\hline \multicolumn{2}{c}{ Species } \\
\hline 26. & Calonotos tiburtus (Cramer, [1779]) \\
27. & Calonotos triplaga (Hampson, 1909) \\
& \\
28. & \\
29. & Chrostosoma dhamis Schaus, 1928 \\
30. & Chrostosoma dolens (Walker, 1854) \\
31. & Chrostosoma haematica (Perty, 1834) \\
32. & Chrostosoma tricolor (R. Felder, 1874) \\
33. & Cosmosoma achemon (Fabricius, 1781)
\end{tabular}

34. Cosmosoma ada (Herrich-Schäffer, [1855])

35. Cosmosoma admota (Herrich-Schäffer, [1854])

36. Cosmosoma anoxanthia Druce, 1905

37. Cosmosoma auge (Linnaeus, 1758)

38. Cosmosoma batesii (Butler, 1876)

39. Cosmosoma centralis (Walker, 1854) AMz

40. Cosmosoma consolata (Walker, 1856)

41. Cosmosoma contracta (Walker, 1856)

42. Cosmosoma corvica (Dognin, 1910)

43. Cosmosoma durca Schaus, $1896^{\mathrm{AMZ}}$

44. Cosmosoma festiva Walker, 1854

45. Cosmosoma klagesi Rothschild, 1910

46. Cosmosoma melathoracia Kaye, 1901

47. Cosmosoma metallescens (Ménétriés, 1857)

48. Cosmosoma nelea Möschler, 1877

49. Cosmosoma pheres (Stoll, [1782]) ${ }^{\mathrm{AMZ}}$

50. Cosmosoma rasera Jones, 1914 AMz

51. Cosmosoma remota (Walker, 1854) ${ }^{\mathrm{AMZ}}$

\section{Record}

AM, Manaus*

AM, Amazons (Hampson 1898), Manaus (Hampson 1909); AP, Serra do Navio*; MA, Açailândia*; PA, Altamira \{Monte Santo\} (Teston \& Delfina 2010) and \{Serra do Pardo National Park\} (Teston \& Correa 2015), Oriximiná \{Rio Cuminá\}*, Parauapebas \{Serra Norte, Carajás ${ }^{*}$, Santarém \{Taperinha\} (Zerny 1931), São Félix do Xingu \{Serra do Pardo National Park\} (Teston \& Correa 2015); RO, Cacaulândia*

PA, Belterra \{National Forest of Tapajós\} (Freitas 2014)

PA, [Belém] (Schaus 1928)

PA, [Belém] (Walker 1854a)

AM, Amazon river (Perty 1834), Benjamin Constant*, São Paulo de Olivença*, Tefé \{Ega\} (Walker [1865]); AP, Serra do Navio*; PA, Belém*, Viseu*

AM, Amazon river (R. Felder 1874)

AM, Benjamin Constant*, Manaus*; MA, Açailândia*; MT, Aripuanã*; PA, [Belém] (Druce 1897), Óbidos*, Prainha (Butler 1878), Santarém (Hampson 1898) \{Taperinha\} (Zerny 1931); RO, Porto Velho*

[AM], Amazonas (Zerny 1912)

PA, Belterra \{National Forest of Tapajós\} (Freitas 2014), Santarém (Valente et al. 2018), São Félix do Xingu \{Serra do Pardo National Park\} (Teston \& Correa 2015)

PA (Hagmann 1938)

AM, Manicoré*; MA, Açailândia*; PA, Santarém \{Mojú and Taperinha\} (Zerny 1931) RO, Candeias do Jamari*

PA, [Belém] (Butler 1876), Santarém \{Taperinha\} (Zerny 1931)

MA, Açailândia*; PA, Belém*; RO, Cacaulândia*

PA, Belém*, Altamira \{Monte Santo\} (Delfina \& Teston 2013), Parauapebas \{Serra Norte, Carajás $\}^{*}$, São Félix do Xingu \{Serra do Pardo National Park\} (Teston \& Correa 2015); RO, Cacaulândia*, Porto Velho*

[AC], Upper Juruá river (Zerny 1931); AM, [Lábrea] \{Rio Purus, Huitanaã (Bryk 1953), Valley of Amazon [river] (Walker 1856); PA, Santarém \{Taperinha\} (Zerny 1931)

PA, Santarém \{Taperinha\} (Zerny 1931), Altamira \{Monte Santo\} (Delfina \& Teston 2013)

MA, Açailândia*

AM, Parintins \{Villa Nova\} (Hampson 1914); MA, Açailândia*

PA, [Belém] (Hampson 1914), São Félix do Xingu \{Serra do Pardo National Park\} (Teston \& Correa 2015)

PA, Santarém \{Taperinha\} (Zerny 1931)

AM, [Autazes] \{Rio Autaz\} (Bryk 1953), [Eirunepé] \{Juruá river, São Felipe\} (Zerny 1931), Parintins \{Villa Nova (Hampson 1898); PA, Altamira $\left\{51^{\circ} \mathrm{BIS}\right\}$ (Teston et al. 2012) and \{Monte Santo $\}$ (Delfina \& Teston 2013) and $\{$ Serra do Pardo National Park (Teston \& Correa 2015), [Belém] (Butler 1876), Belterra \{National Forest of Tapajós\} (Freitas 2014), Marabá*, Santarém \{Taperinha\} (Zerny 1931), São Félix do Xingu \{Serra do Pardo National Park\} (Teston \& Correa 2015)

PA, São Félix do Xingu \{Serra do Pardo National Park\} (Teston \& Correa 2015)

AM, Manaus*; MA, Açailândia*; PA, Belém*; RO, Cacaulândia*

MA, Açailândia*; PA, Marabá*, Novo Progresso \{Cachimbo\}*; RO, Candeias do Jamari*

AM, Manicoré*, Santa Isabel do Rio Negro*; MA, Açailândia*; MT, Aripuanã*; PA, Belém*, Capitão Poço*, Parauapebas $\{$ Serra Norte, Carajás $\} *$, Santarém*, Viseu*; RO, Cacaulândia* 
Continuation Table 1.

\begin{tabular}{cl}
\hline \multicolumn{2}{c}{ Species } \\
\hline 52. & $\begin{array}{l}\text { Cosmosoma seraphina } \text { (Herrich-Schäffer, } \\
{[1854] \text { ) }}\end{array}$ \\
53. & Cosmosoma stibosticta (Butler, 1876) \\
54. & Cosmosoma subflamma (Walker, 1854)
\end{tabular}

55. Cosmosoma telephus (Walker, 1854)

\section{Record}

PA, [Belém] (Herrich-Schäffer [1854]), Novo Progresso \{Cachimbo\}*; RO, Porto Velho*

PA, Altamira \{Monte Santo\} (Teston \& Delfina 2010)

AM, Amazonas (Zerny 1931), Manaus*, Rio Urubu*; PA, Altamira \{Monte Santo\} (Teston \& Delfina 2010), Belém*, Bragança*, Itaituba*, Santarém \{Taperinha\} (Zerny 1931), São Félix do Xingu \{Serra do Pardo National Park\} (Teston \& Correa 2015)

AM, [Eirunepé] \{Juruá river, São Felipe\} (Zerny 1931); AP, Serra do Navio*; MA, Açailândia*; MT, Aripuanã*, Sinop*; PA, Altamira \{Serra do Pardo National Park\} (Teston \& Correa 2015), Belém*, Novo Progresso \{Cachimbo\}*, Parauapebas \{Serra Norte, Carajás\}*, Peixe Boi*, Santarém \{Taperinha\} (Zerny 1931), São Félix do Xingu \{Serra do Pardo National Park\} (Teston \& Correa 2015); RO, Cacaulândia*

PA, [Belém] (Hampson 1898)

MA, Açailândia*; MT, Aripuanã*; PA, Altamira \{Serra do Pardo National Park\} (Teston \& Correa 2015), Parauapebas \{Serra Norte, Carajás $\}$ *, Santarém (Butler 1876) \{Taperinha\} (Zerny 1931), São Félix do Xingu \{Serra do Pardo National Park\} (Teston \& Correa 2015); RO, Cacaulândia*

PA, Santarém \{Taperinha\} (Zerny 1931), Altamira \{Monte Santo\} (Teston \& Delfina 2010), São Félix do Xingu \{Serra do Pardo National Park\} (Teston \& Correa 2015)

AM, [Boa Vista do Ramos] \{Massauari\} (Hampson 1898)

AM, Amazons (Rothschild 1910), Benjamin Constant*, Fonte Boa (Rothschild 1910), Manicoré*, São Paulo de Olivença (Hampson 1901), Tefé (Rothschild 1910); PA, Óbidos*, Santarém*; RO, Cacaulândia*, Porto Velho \{Aliança\} (Rothschild 1910)

AM, Tefé \{Ega\} (Walker [1865]); PA, Santarém \{Taperinha\} (Zerny 1931)

[RR], [Caracaraí] \{Rio Branco\} (Bryk 1953)

AM, Fonte Boa*, Manaus*, Rio Urubu*; MA, Açailândia*; PA, Altamira \{Monte Santo $\}$ (Teston \& Delfina 2010) $\left\{51^{\circ}\right.$ BIS $\}$ (Teston et al. 2012), Belém*, Belterra \{National Forest of Tapajós\} (Freitas 2014), Chaves (Hampson 1898), Santarém (Valente et al. 2018), São Félix do Xingu \{Serra do Pardo National Park\} (Teston \& Correa 2015), Soure*

PA, São Félix do Xingu \{Serra do Pardo National Park\} (Teston \& Correa 2015) AM, Amazon river (Perty 1834)

AM, Benjamin Constant*; MA, Açailândia*; PA, Marabá*

[AM], Amazons (Rothschild 1931)

AM, Tefé $\{$ Ega $\}$ (Walker [1865])

AM, Amazon river (Rothschild 1911)

AM, Amazon river (Perty 1834), Parintins \{Villa Nova\} (Walker 1856)

RO, Cacaulândia*

AM, São Paulo de Olivença*

AM, Benjamin Constant (Rio Javari) (Machado Filho \& Rêgo Barros 1973), [Beruri] $\{\operatorname{Re}[\mathrm{d}] \mathrm{e}[\mathrm{n}][$ ção] on Rio Purus $\}$ (Butler 1878), Borba (Machado Filho \& Rêgo Barros 1973), [Ipixuna] \{Lago Cerrado on Rio Juruá\} (Butler 1878), Manaus (Zerny 1931), Parintins \{Villa Nova\} (Butler 1876), São Paulo [de Olivença] \{Solimões [river]\} (Bryk 1953), Tabatinga (Butler 1878), Tefé \{Ega\} (Butler 1876); PA, Óbidos (Machado Filho \& Rêgo Barros 1973), Santarém \{Tapará\} (Zerny 1931)

AM, Manicoré \{Rio Madeira\} (Machado Filho \& Rêgo Barros 1973)

AM, Benjamin Constant*, [Maués] \{Rio Para[u]ar[i]\} (Machado Filho \& Rêgo Barros 1973), São Paulo de Olivença (Machado Filho \& Rêgo Barros 1973), Tefé (Machado Filho \& Rêgo Barros 1973); AP, Serra do Navio (ICOMI) (Machado Filho \& Rêgo Barros 1973); PA, [Almeirim] \{Rio Paru $\}^{*}$, Altamira $\left\{51^{\circ}\right.$ BIS $\}$ (Teston et al. 2012), Marabá*, Óbidos*, Oriximiná \{Rio Cuminá\}*, Parauapebas \{Serra Norte, Carajás $\}^{*}$, Santarém \{Taperinha\} (Draudt 1931) 
Teston \& Ferro

Continuation Table 1.

\begin{aligned} & \hline \multicolumn{2}{c}{ Species } \\ & \hline 76. Histioea glaucozona Druce, 1898 \\ & 77. Histioea hoffmannsi Rothschild, 1911 \\ & 78. Histioea paraensis Machado Filho \& Rêgo \\ & Barros, 1971 \\ & 79. Histioea proserpina (Hübner, 1827) \end{aligned}

80. Homoeocera stictosoma Druce, 1898

81. Hyda basilutea (Walker, 1854)

82. Hypatia delecta (Butler, 1876)

83. Hypatia melaleuca (Walker, 1854)

84. Hypocharis albicincta Cerda, 2008

85. Hypocharis clusia (Druce, 1897)

86. Isanthrene aterrima (Walker, [1865])

87. Isanthrene melas (Cramer, [1775])

88. Isanthrene notipennis (Butler, 1876)

89. Isanthrene porphyria (Walker, 1854)

90. Isanthrene profusa Hampson, 1898

91. Isanthrene varia (Walker, 1854)

92. Isanthrene vespiformes (Butler, 1876)

93. Lepidoneiva erubescens (Butler, 1876)

94. Leucotmemis climacina (Butler, 1876) ${ }^{\mathrm{NEW}}$

95. Leucotmemis dorsalis (Walker, 1854)

96. Leucotmemis emergens (Walker, [1865])

97. Leucotmemis felderi (Rothschild, 1911)

98. Leucotmemis flavidior Gaede, 1926

\section{Record}

[AM], Amazons (Druce 1898b)

AM, Humaitá \{Rio Madeira\} (Rothschild 1911)

PA, Óbidos (Machado Filho \& Rêgo Barros 1971)

AC, Porto Walter \{Alto Juruá\} (Machado Filho \& Rêgo Barros 1971), Xapuri (Machado Filho \& Rêgo Barros 1971); AM, Amazons (Hampson 1898), [Autazes] \{Rio Autaz\} (Bryk 1953), Benjamin Constant \{Rio Javari\} (Machado Filho \& Rêgo Barros 1971), [Eirunepé] \{Juruá river, São Felipe\} (Zerny 1931), Manaus (Machado Filho \& Rêgo Barros 1971), Maués*, Rio Negro (Zerny 1931), São Paulo de Olivença (Machado Filho \& Rêgo Barros 1971), São Gabriel da Cachoeira*, Tefé \{Egas\} (Machado Filho \& Rêgo Barros 1971); PA, Óbidos (Machado Filho \& Rêgo Barros 1971), Oriximiná \{Rio Cuminá\}*, Santarém \{Taperinha\} (Zerny 1931)

PA, Almeirim \{Jari\} (Hawes et al. 2009)

AM, Santa Isabel do Rio Negro*; AP, Mazagão*; MT (Zerny 1931), Sinop*; PA, Belém*, Igarapé Açú*, Ourém*, Prainha (Butler 1878), Santarém \{Taperinha\} (Zerny 1931), Tucuruí*

PA, [Belém] (Butler 1876)

PA, [Belém] (Walker 1854a); RO, Cacaulândia*

PA, Belterra \{National Forest of Tapajós\} (Freitas 2014)

AM, São Paulo de Olivença*; PA (Hagmann 1938)

AM, Parintins*, Tefé \{Ega\} (Walker [1865]); AP, Serra do Navio*; RO, Cacaulândia*

AM, Benjamin Constant*, [Eirunepé] \{Juruá river, Matto Pyri\} (Zerny 1931), Parintins \{Villa Nova\} (Hampson 1898), São Paulo de Olivença*; PA, [Belém] (Walker 1854a), Óbidos (Zerny 1931), Santarém \{Taperinha\} (Zerny 1931), São Félix do Xingu \{Serra do Pardo National Park\} (Teston \& Correa 2015)

AM, Parintins \{Villa Nova\} (Butler 1876); MA, Açailândia*; PA, Óbidos*, Santarém*

AM, Benjamin Constant*, [Eirunepé] \{Juruá river, São Felipe and Matto Pyri\} (Zerny 1931), Manicoré*, Parintins \{Villa Nova\} (Hampson 1898), São Paulo de Olivença*, Tefé $\{$ Ega $\}$ (Hampson 1898); AP, Serra do Navio*; MA, Açailândia*; PA, Altamira \{Serra do Pardo National Park\} (Teston \& Correa 2015), [Belém] (Hampson 1898), Paragominas*, Santarém*, São Félix do Xingu \{Serra do Pardo National Park $\}$ (Teston \& Correa 2015); RO, Cacaulândia*, Jarú*

AM, Benjamin Constant*, Tefé \{Ega\} (Hampson 1898); PA, Altamira \{Monte Santo $\}$ (Teston \& Delfina 2010) and $\left\{51^{\circ}\right.$ BIS $\}$ (Teston et al. 2012) and \{Serra do Pardo National Park (Teston \& Correa 2015), São Félix do Xingu \{Serra do Pardo National Park\} (Teston \& Correa 2015)

AM, São Paulo de Olivença*; MT, Sinop*; PA, Altamira \{Serra do Pardo National Park (Teston \& Correa 2015), [Belém] (Walker 1854a), Santarém \{Mojú and Taperinha\} (Zerny 1931), São Félix do Xingu \{Serra do Pardo National Park\} (Teston \& Correa 2015); RO, Porto Velho*

AM, Parintins \{Villa Nova\} (Butler 1876); PA, Itaituba*, Óbidos (Zerny 1931), Santarém \{Taperinha\} (Zerny 1931), São Félix do Xingu \{Serra do Pardo National Park $\}$ (Teston \& Correa 2015)

AM (Bryk 1953); MT, Sinop*; PA, Novo Progresso $\{\text { Cachimbo }\}^{*}$

MA, Açailândia*; RO, Cacaulândia*

AM, Manaus (Bryk 1953); MA, Açailândia*; PA, Santarém (Walker 1854a); RO, Cacaulândia*

AM, Manaus (Bryk 1953), Tefé \{Ega\} (Walker [1865])

AM, Amazon river (Rothschild 1911)

PA, [Belém] (Gaede 1926) 
Continuation Table 1.

\begin{aligned} & \hline Species \\ & \hline 99. Leucotmemis intersecta (Walker, [1865]) \\ & 100. Leucotmemis margariphera (Butler, 1876) \\ & 101. Leucotmemis nexa (Herrich-Schäffer, [1854]) \\ & \\ & 102. Leucotmemis ornatula (Walker, 1854) \\ & 103. Leucotmemis tenthredoides (Walker, 1856) \end{aligned}

104. Leucotmemis torrida (Walker, 1854)

105. Leucotmemis varipes (Walker, 1854)

106. Loxophlebia cinctata Hampson, 1905

107. Loxophlebia crocata (Herrich-Schäffer, [1854]) AMz

108. Loxophlebia crusmatica Dognin, 1911

109. Loxophlebia diaphana (Sepp, [1848])

110. Loxophlebia imitata (Druce, 1884) AMZ $^{\mathrm{M}}$

111. Loxophlebia picta (Walker, 1854)

112. Loxophlebia postflavia Druce, 1898

113. Loxophlebia pyrgion (Druce, 1884)

114. Loxophlebia roseipectus Rothschild, 1931

115. Loxophlebia semiaurantia Rothschild, 1931

116. Loxophlebia triangulifera (R. Felder, 1874)

117. Macrocneme adonis Druce, 1884

118. Macrocneme chrysitis (Guérin-Méneville, [1844])

119. Macrocneme lades (Cramer, [1775])

120. Macrocneme leucostigma (Perty, 1834)

121. Macrocneme maja (Fabricius, 1787)

\section{Record}

AM, Tefé \{Ega\} (Walker [1865]); PA (Hagmann 1938)

PA, [Belém] (Butler 1876), São Félix do Xingu \{Serra do Pardo National Park\} (Teston \& Correa 2015)

AM, Parintins \{Villa Nova\} (Butler 1876); AP, Serra do Navio*; MT, Sinop*; PA, Altamira \{Serra do Pardo National Park\} (Teston \& Correa 2015), Belterra \{National Forest of Tapajós\} (Freitas 2014), Óbidos (Zerny 1931), Santarém (Herrich-Schäffer [1854]) \{Taperinha\} (Zerny 1931), São Félix do Xingu \{Serra do Pardo National Park\} (Teston \& Correa 2015); RO, Cacaulândia*

PA, [Belém] (Walker 1854a)

AM, Rio Juruá (Butler 1878), Santa Isabel do Rio Negro*, Tefé \{Ega\} (Butler 1876), Tonantins (Bryk 1953), Valley of the Amazons [river] (Walker 1856); PA, Belém*, Belterra \{National Forest of Tapajós\} (Freitas 2014), Santarém \{Taperinha\} (Zerny 1931); RO, Porto Velho*

PA, Altamira \{Monte Santo\} (Teston \& Delfina 2010), Belterra \{National Forest of Tapajós\} (Freitas 2014), Santarém \{Taperinha\} (Zerny 1931), Tapajós [river] (Walker 1854a)

AM, Manaus (Butler 1878); PA, [Belém] (Walker 1854a), Belterra \{National Forest of Tapajós\} (Freitas 2014), Santarém \{Taperinha\} (Zerny 1931); RO, Cacaulândia*

AM, Barcelos \{Moura and Tomar\}*, Manaus*; MT, Sinop*; PA, Santarém \{Taperinha\} (Zerny 1931); RO, Cacaulândia*

MA, Açailândia*; PA, Capitão Poço*

PA (Hagmann 1938)

PA, [Belém] (Walker 1854a), Santarém \{Taperinha\} (Zerny 1931), São Félix do Xingu \{Serra do Pardo National Park\} (Teston \& Correa 2015)

MA, Açailândia*

AM, Santa Isabel do Rio Negro*; MA, Açailândia*; PA, [Belém] (Walker 1854a), Capitão Poço*, Novo Progresso \{Cachimbo\}*, Santarém (Walker 1854a) \{Taperinha\} (Zerny 1931); RO, Cacaulândia*, Porto Velho*

PA, Santarém \{Taperinha\} (Zerny 1931)

PA, Altamira \{Serra do Pardo National Park\} (Teston \& Correa 2015), Santarém \{Taperinha\} (Zerny 1931), São Félix do Xingu \{Serra do Pardo National Park\} (Teston \& Correa 2015)

AM, [Itacoatiara] \{Lower Amazons, junction with Rio Madeira\} (Rothschild 1931)

PA, [Belém] (Rothschild 1931)

[AM], Amazons (Hampson 1898); PA, São Félix do Xingu \{Serra do Pardo National Park\} (Teston \& Correa 2015)

AM, Barcelos \{Moura\}*, Borba*, Manaus*, São Paulo de Olivença*; PA (Hagmann 1938), Belém*, Belterra \{National Forest of Tapajós\} (Freitas 2014), Santarém (Valente et al. 2018), São Félix do Xingu \{Serra do Pardo National Park\} (Teston \& Correa 2015)

AM, Rio Purus (Bryk 1953); PA, Santarém \{Taperinha\} (Zerny 1931)

AM, Manicoré*; PA, Altamira \{Monte Santo\} (Teston \& Delfina 2010) and \{Serra do Pardo National Park $\}$ (Teston \& Correa 2015), [Belém] (Hampson 1898), Belterra \{National Forest of Tapajós\} (Freitas 2014), Capitão Poço*, Santarém (Valente et al. 2018), São Félix do Xingu \{Serra do Pardo National Park\} (Teston \& Correa 2015)

PA, Santarém \{Taperinha\} (Zerny 1931)

AM, Rio Negro (Zerny 1931); PA, [Belém] (Hampson 1898), Santarém \{Taperinha\} (Zerny 1931) 
Teston \& Ferro

Continuation Table 1.

\begin{aligned} \hline & Species \\ \hline 122. & Macrocneme thyra Möschler, 1883 \\ 123. & Macrocneme thyridia Hampson, 1898 \\ 124. & Macrocneme verdivittata (Klages, 1906) \\ & \\ 125. & Macrocneme vidua (Bryk, 1953) \\ 126. & Macrocneme zongonata Dietz, 1994 \\ 127. & Mesothen desperata (Walker, 1856) \end{aligned}

128. Mesothen endoleuca Druce, 1905

129. Mesothen inconspicuata (Kaye, 1911) AMZ

130. Mesothen pyrrha Schaus, 1889

131. Metaloba argante (Druce, 1897)

132. Metamya chrysonota (Hampson, 1898)

133. Metamya intersecta (Hampson, 1898)

134. Metamya picta (Druce, 1898) AMZ

135. Methysia notabilis (Walker, 1854)

136. Micragyrta diminuta (Walker, 1854)

137. Mimagyrta abdominalis (Rothschild, 1912)

138. Mimagyrta pampa (Druce, 1893)

139. Mystrocneme atavia Hampson, 1898

140. Mystrocneme varipes (Walker, 1854)

141. Nyridela chalciope (Hübner, 1827)

142. Orcynia calcarata (Walker, 1854)

143. Pezaptera sordida (Walker, 1856)

144. Phaeosphecia opaca (Walker, 1856)

145. Pheia admirabilis Bryk, 1953

146. Pheia albisigna (Walker, 1854)

147. Pheia elegans (Druce, 1884) ${ }^{\mathrm{AMZ}}$

\section{Record}

AM, São Paulo de Olivença*, Tefé (Bryk 1953); PA, [Belém] (Hampson 1898), Belterra \{National Forest of Tapajós\} (Freitas 2014)

AM (Travassos Filho 1940); PA, Santarém (Valente et al. 2018)

AM, Barcelos $\{\text { Moura }\}^{*}$; PA, Novo Progresso $\{$ Cachimbo $\} *$, Parauapebas $\{$ Serra Norte, Carajás $\}^{*}$

[RR], [Caracaraí] \{Rio B[r]anco\} (Bryk 1953)

PA, Santarém (Valente et al. 2018)

AM, Valley of [river] Amazon (Walker 1856); PA (Hagmann 1938), Altamira \{Serra do Pardo National Park\} (Teston \& Correa 2015), Belterra \{National Forest of Tapajós\} (Freitas 2014), Santarém*, São Félix do Xingu \{Serra do Pardo National Park\} (Teston \& Correa 2015)

PA, Santarém \{Taperinha\} (Zerny 1931)

RO, Cacaulândia*; PA, Capitão Poço*

PA, Santarém \{Taperinha\} (Zerny 1931)

MA, Açailândia*; PA, Altamira \{Monte Santo\} (Teston \& Delfina 2010) and \{Serra do Pardo National Park\} (Teston \& Correa 2015), Belterra \{National Forest of Tapajós\} (Freitas 2014), Novo Progresso \{Cachimbo\}*, Parauapebas \{Serra Norte, Carajás $\}^{*}$, São Félix do Xingu \{Serra do Pardo National Park\} (Teston \& Correa 2015); RO, Cacaulândia*

PA, Itaituba (Hampson 1898)

PA, [Belém] (Hampson 1898)

AM, Manicoré*; PA, Santarém*

PA, [Belém] (Walker 1854a), Santarém*

AM, Borba*, Tefé \{Ega\} (Walker [1865]); PA, [Belém] (Walker 1854b), Santarém \{Taperinha\} (Zerny 1931)

AM, Humaitá (Rothschild 1912); PA, [Belém] (Zerny 1931)

AM, Tefé (Hampson 1898)

AM, Fonte Boa (Hampson 1898)

AM, Santa Isabel do Rio Negro*, Tefé*; PA, Anajás*, [Belém] (Walker 1854a), Breves*, Cametá (Zerny 1931)

AM, Manaus*; PA, Altamira \{Serra do Pardo National Park\} (Teston \& Correa 2015), Belém*, Capitão Poço*, Novo Progresso \{Cachimbo ${ }^{*}$, Óbidos*, Parauapebas $\left\{\right.$ Serra Norte, Carajás ${ }^{*}$, Santarém $\{$ Taperinha\} (Zerny 1931), São Félix do Xingu \{Serra do Pardo National Park\} (Teston \& Correa 2015); RO, Cacaulândia*

AM, Manaus*, Novo Aripuanã*, Tefé*; AP, Serra do Navio*; MA, Açailândia*; PA, Almeirim \{Jari\} (Hawes et al. 2009), Belém \{Utinga\} (Pereira 1958), Marabá*, Novo Progresso \{Cachimbo\} (Pereira 1958), Parauapebas \{Serra Norte, Carajás\}*, Santarém (Walker 1854a) \{Taperinha\} (Zerny 1931), São Félix do Xingu \{Serra do Pardo National Park $\}$ (Teston \& Correa 2015); RO, Cacaulândia*, Jarú*

AM, Manaus (Zerny 1931), Parintins \{Villa Nova\} (Hampson 1898), Tefé $\{$ Ega\} (Walker [1865]); PA, Santarém (Walker 1856)

MA, Açailândia*; PA, [Belém] \{Pará, Valley of the Amazon [river]\} (Walker 1856), Santarém*

AM, [São Gabriel da Cachoeira] \{Taracuá\} (Bryk, 1953)

AM, Tefé (Hampson 1898); MT, Sinop*; PA, Novo Progresso \{Cachimbo $\}^{*}$, Parauapebas $\left\{\right.$ Serra Norte, Carajás ${ }^{*}$, Santarém $\{$ Taperinha\} (Zerny 1931), São Félix do Xingu \{Serra do Pardo National Park\} (Teston \& Correa 2015); RO, Cacaulândia*, Porto Velho*; [RR], [Caracaraí] \{Rio Branco\} (Bryk 1953) 
Continuation Table 1.

\begin{tabular}{ll}
\hline 148. & Pheia gaudens (Walker, 1856) \\
\hline 149. & Pheia haemapleura Hampson, 1914 \\
150. & Pheia haematosticta Jones, 1908 AMz \\
151. & Pheia serpensis Kaye, 1918 \\
152. & Pheia sperans (Walker, 1856) \\
153. & Pheia taperinhae Dognin, 1923 \\
154. & Pheia utica (Druce, 1889) \\
155. & Phoenicoprocta corvica (Dognin, 1910)
\end{tabular}

156. Phoenicoprocta haemorrhoidalis (Fabricius, 1775) AMZ

157. Phoenicoprocta insperata (Walker, 1856)

158. Phoenicoprocta sanguinea (Walker, 1854) NEW

159. Phoenicoprocta vacillans (Walker, 1856)

160. Pleurosoma angustata (Moeschler, 1878)

161. Poecilosoma chrysis Hübner, 1823

162. Poecilosoma eone (Hübner, 1827)

163. Poecilosoma marginatum (Walker, 1856)

164. Poecilosoma nigerrima (Walker, [1865])

165. Poliopastea anthracina (Klages, 1906)

166. Poliopastea coelebs Bryk, 1953

167. Poliopastea errans (Hübner, [1819])

168. Poliopastea esmeralda (Butler, 1876)

169. Poliopastea indistincta (Butler, 1876)

170. Poliopastea plumbea Hampson, 1898

171. Poliopastea vittata (Walker, 1854)

172. Pompiliodes aliena (Walker, 1854)

173. Pompiliodes postica (Walker, 1856)

\section{Record}

PA, [Belém] (Walker 1856), Belterra \{National Forest of Tapajós\} (Freitas 2014), Santarém \{Taperinha\} (Zerny 1931), São Félix do Xingu \{Serra do Pardo National Park (Teston \& Correa 2015); RO, Cacaulândia*

PA (Hagmann 1938)

PA, Novo Progresso \{Cachimbo * $^{*}$, Santarém*; RO, Cacaulândia*

AM, Itacoatiara \{Serpa\} (Kaye 1918); PA, Santarém \{Taperinha\} (Zerny 1931)

[AM], Valley of the Amazon [river] (Walker 1856)

PA, Santarém \{Taperinha\} (Dognin 1923)

PA, Santarém (Valente et al. 2018)

PA, Altamira \{Serra do Pardo National Park\} (Teston \& Correa 2015), Belterra \{National Forest of Tapajós\} (Freitas 2014), Santarém (Valente et al. 2018), São Félix do Xingu \{Serra do Pardo National Park\} (Teston \& Correa 2015)

MA, Açailândia*; PA, Capitão Poço*

AM, [Atalaia do Norte] \{Braga on Rio Javary\} (Butler 1877), Santa Isabel do Rio Negro*, São Paulo de Olivença*; PA, [Belém] (Walker 1856), Santarém \{Taperinha\} (Zerny 1931)

AM, Manaus*, São Paulo de Olivença*; AP, Serra do Navio*; PA, Belém*, Santarém*

AM, Benjamin Constant*, Manaus*, [São Gabriel da Cachoeira] \{Taracuá\} (Bryk, 1953), São Paulo de Olivença*, Tefé*; AP, Serra do Navio*; PA, Altamira \{Monte Santo (Teston \& Delfina 2010), Belterra \{National Forest of Tapajós\} (Freitas 2014), Santarém \{Taperinha and Moju\} (Zerny 1931), São Félix do Xingu \{Serra do Pardo National Park\} (Teston \& Correa 2015); RO, Porto Velho*

PA, Santarém \{Taperinha\} (Zerny 1931), São Félix do Xingu \{Serra do Pardo National Park $\}$ (Teston \& Correa 2015)

AM, Tefé \{Ega\} (Walker [1865]); PA, Belém*, Breves (Zerny 1931), Marabá*, Santarém \{Taperinha\} (Zerny 1931), São Félix do Xingu \{Serra do Pardo National Park\} (Teston \& Correa 2015); RO, Cacaulândia*

AM, [Eirunepé] \{Juruá river, São Felipe\} (Zerny 1931), Itacoatiara (Zerny 1931), Manaus (Zerny 1931), Tefé $\{$ Ega $\}$ (Walker 1854a); PA, Altamira $\left\{51^{\circ}\right.$ BIS $\}$ (Teston et al. 2012) and \{Serra do Pardo National Park\} (Teston \& Correa 2015), Santarém (Walker 1854a) \{Taperinha\} (Zerny 1931), São Félix do Xingu \{Serra do Pardo National Park\} (Teston \& Correa 2015)

AM, Valley of the Amazon [river] (Walker 1856)

AM, Tefé $\{$ Ega $\}$ (Walker [1865])

PA, Altamira \{Monte Santo\} (Delfina \& Teston 2013), Belém*, Belterra \{National Forest of Tapajós\} (Freitas 2014), Santarém \{Taperinha\} (Zerny 1931), São Félix do Xingu \{Serra do Pardo National Park\} (Teston \& Correa 2015)

AM, Amazonas [river] (Bryk 1953)

PA, [Belém] (Butler 1876)

AM, Tefé $\{$ Ega\} (Butler 1876)

AM, Alto Amazonas (Hagmann 1938); PA, [Belém] (Butler 1876); RO, Cacaulândia*

AM, Parintins (Hampson 1898), Tabatinga (Hampson 1898); PA, Altamira \{Monte Santo\} (Teston \& Delfina 2010) and \{Serra do Pardo National Park\} (Teston \& Correa 2015), Belém*, Santarém \{Taperinha\} (Zerny 1931), São Félix do Xingu \{Serra do Pardo National Park\} (Teston \& Correa 2015)

PA, [Belém] (Walker 1854a), Santarém \{Taperinha and Moju\} (Zerny 1931)

AM, Manaus (Hampson 1898), Tefé \{Ega\} (Hampson 1898); MA, Açailândia*; PA, [Belém] (Walker 1854a), Santarém \{Taperinha\} (Zerny 1931); RO, Cacaulândia*

PA, Santarém (Walker 1856) \{Taperinha\} (Zerny 1931) 
Teston \& Ferro

Continuation Table 1.

\begin{tabular}{ll}
\hline & Species \\
\hline 174. & Pompiliodes tenebrosa (Walker, 1854) \\
175. & Pompilopsis tarsalis (Walker, 1854) \\
& \\
176. & Pseudomya picta Schaus, 1894 \\
177. & Psoloptera leucosticta (Hubner, 1827)
\end{tabular}

178. Psoloptera leucotmemica Bryk, 1953

179. Psoloptera melini Bryk, 1953

180. Psoloptera thoracica (Walker, 1854)

181. Rhynchopyga discalba Kaye, 1918

182. Rhynchopyga meisteri (Berg, 1883)

183. Rhynchopyga pimpinella Bryk, 1953

184. Sarosa acutior (R. Felder, 1869)

185. Sarosa ignicornis Hampson, 1914

186. Sarosa mora Schaus, 1911 NEW

187. Sarosa pompilina Butler, $1876^{\mathrm{AMZ}}$

188. Saurita attenuata Hampson, 1905

189. Saurita biradiata (R. Felder, 1869)

190. Saurita cassandra (Linnaeus, 1758)

191. Saurita concisa (Walker, 1854)

192. Saurita concisina Bryk, 1953

193. Saurita cryptoleuca (Walker, 1854)

194. Saurita fumosa (Schaus, 1912) NEw

195. Saurita fusca Dognin, 1923

196. Saurita intricata (Walker, 1854) NEW

197. Saurita lacteata (Butler, 1877)

198. Saurita lasiphlebia Dognin, 1906

199. Saurita melanifera Kaye, 1911

200. Saurita pebasa (Kaye, 1918)

201. Saurita sericea (Herrich-Schäffer, [1854]) AMZ

202. Saurita temenus (Stoll, [1781])

203. Saurita tipulina (Hübner, [1812])

\section{Record}

PA, [Belém] (Walker 1854a)

AM, Manicoré \{Rio Madeira\} (Zerny 1931); PA, [Belém] (Walker 1854a), Santarém \{Taperinha\} (Zerny 1931)

PA, São Félix do Xingu \{Serra do Pardo National Park\} (Teston \& Correa 2015)

MA, Açailândia*; PA, Altamira \{Serra do Pardo National Park\} (Teston \& Correa 2015), [Belém] (Hampson 1898), Maraba*, Santarém (Hampson 1898), São Félix do Xingu \{Serra do Pardo National Park\} (Teston \& Correa 2015); RO, Cacaulândia*, Jarú*

AM, [Autazes] \{Rio Autaz, Curur[ú]\} (Bryk 1953)

AM, Manaus (Bryk 1953)

AM, Benjamin Constant*, Borba*, São Paulo de Olivença*, Tefé $\{$ Ega $\}$ (Walker 1854a); RO, Porto Velho*

PA, Santarém (Valente et al. 2018)

PA, Tapajós [river] (Hampson 1898)

AM, Manaus (Bryk 1953)

AM, Amazonas [river] (R. Felder 1869), Benjamin Constant*; MA, Açailândia*; PA, Belterra \{National Forest of Tapajós\} (Freitas 2014), Capitão Poço*, Marabá*, Novo Progresso \{Cachimbo\}*, Santarém \{Taperinha and Moju\} (Zerny 1931), São Félix do Xingu \{Serra do Pardo National Park\} (Teston \& Correa 2015); RO, Cacaulândia*, Jarú*, Porto Velho*

PA, Santarém \{Taperinha\} (Zerny 1931)

AP, Porto Grande*, Serra do Navio*

AM, Benjamin Constant*; PA, Novo Progresso $\{\text { Cachimbo }\}^{*}$

PA, Altamira \{Serra do Pardo National Park\} (Teston \& Correa 2015), [Oriximiná] \{Rio Trombetas, Rapid of Porteira\} (Butler 1877), São Félix do Xingu \{Serra do Pardo National Park (Teston \& Correa 2015)

AM, Amazonas [river] (R. Felder 1869)

AM, Benjamin Constant*, Fonte Boa*; PA, Altamira \{Monte Santo\} (Teston \& Delfina 2010), [Belém] (Zerny 1931), Belterra \{National Forest of Tapajós\} (Freitas 2014), Benevides*, Breves*, Capitão Poço*, Gurupá*, Marabá*, Ourém*, Santarém (Valente et al. 2018), Tucuruí*; RO, Porto Velho*

PA, [Belém] (Walker 1854a), Belterra \{National Forest of Tapajós\} (Freitas 2014), Santarém (Valente et al. 2018)

AM, Rio Purús (Bryk 1953)

PA, [Belém] (Walker 1854a)

AM, Benjamin Constant*

PA, [Vitória do Xingu] \{Ponte Nova, Rio Xingu\} (Dognin 1923)

RO, Cacaulândia*

AM, Rio Jutaí (Butler 1877); PA, Santarém \{Taperinha\} (Zerny 1931)

AP, Serra do Navio*; PA, Bragança (Oberthür 1912)

AM, Rio Purús (Bryk 1953)

PA, Belterra \{National Forest of Tapajós\} (Freitas 2014)

MA, Açailândia*

AM, Manaus (Bryk 1953), Rio Purús (Bryk 1953), São Gabriel [da Cachoeira] (Bryk 1953); PA, Altamira \{Monte Santo\} (Teston \& Delfina 2010), [Belém] (Walker 1854a), Santarém \{Taperinha\} (Zerny 1931), São Félix do Xingu \{Serra do Pardo National Park\} (Teston \& Correa 2015); [RR], [Caracaraí] \{Rio B[r]anco\} (Bryk 1953)

PA, Altamira $\left\{51^{\circ} \mathrm{BIS}\right\}$ (Teston et al. 2012) \{Monte Santo (Delfina \& Teston 2013), [Belém] (Walker 1854a), Belterra \{National Forest of Tapajós\} (Freitas 2014), São Félix do Xingu \{Serra do Pardo National Park\} (Teston \& Correa 2015) 
Continuation Table 1.

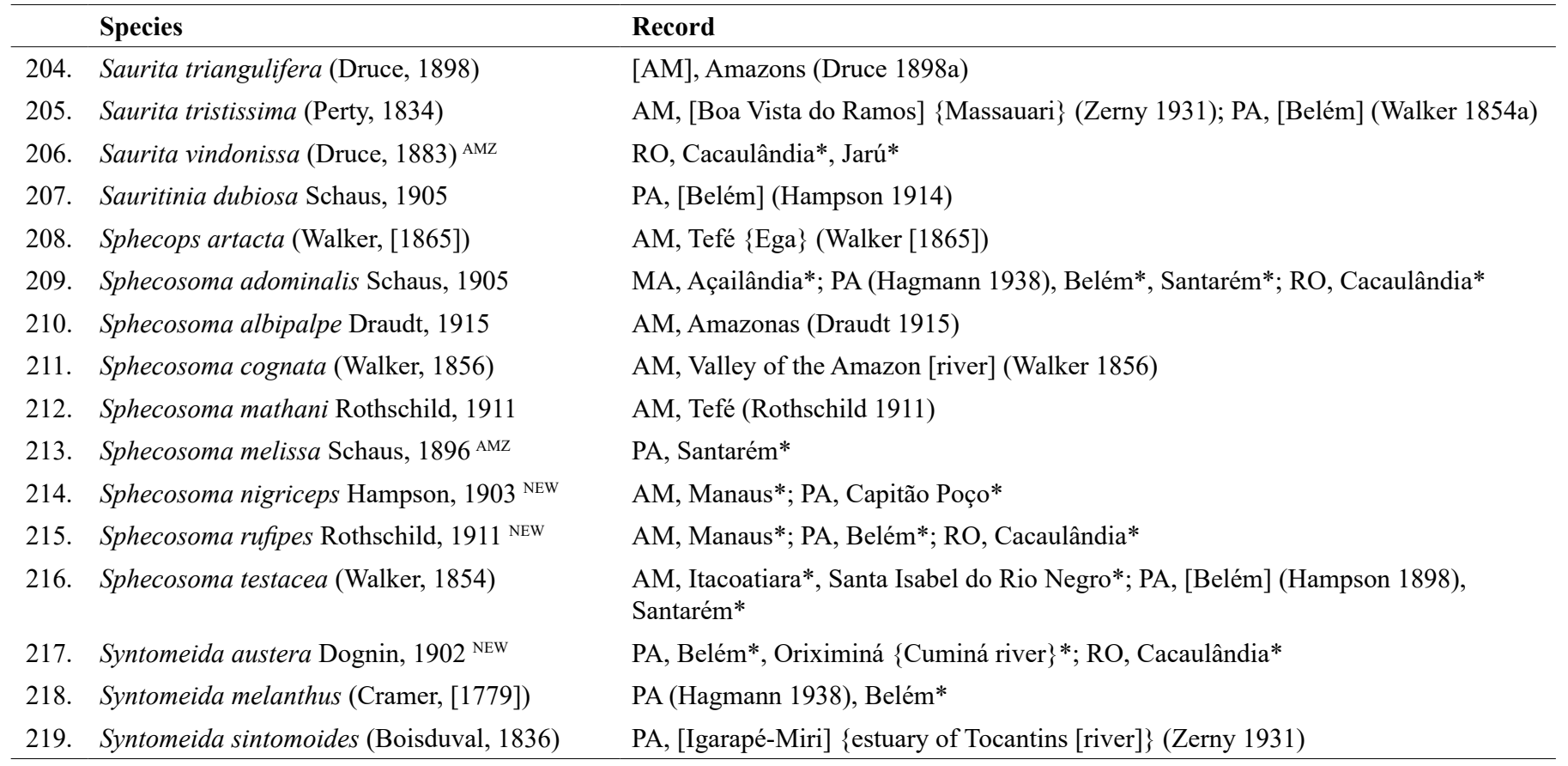

AMZ Species recorded for states within the Amazon biome by Ferro and Diniz (2010), but without precise location and biome information, and Amazon biome by

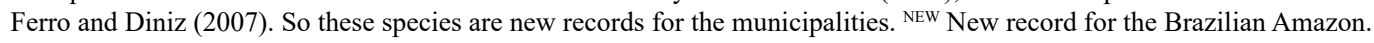

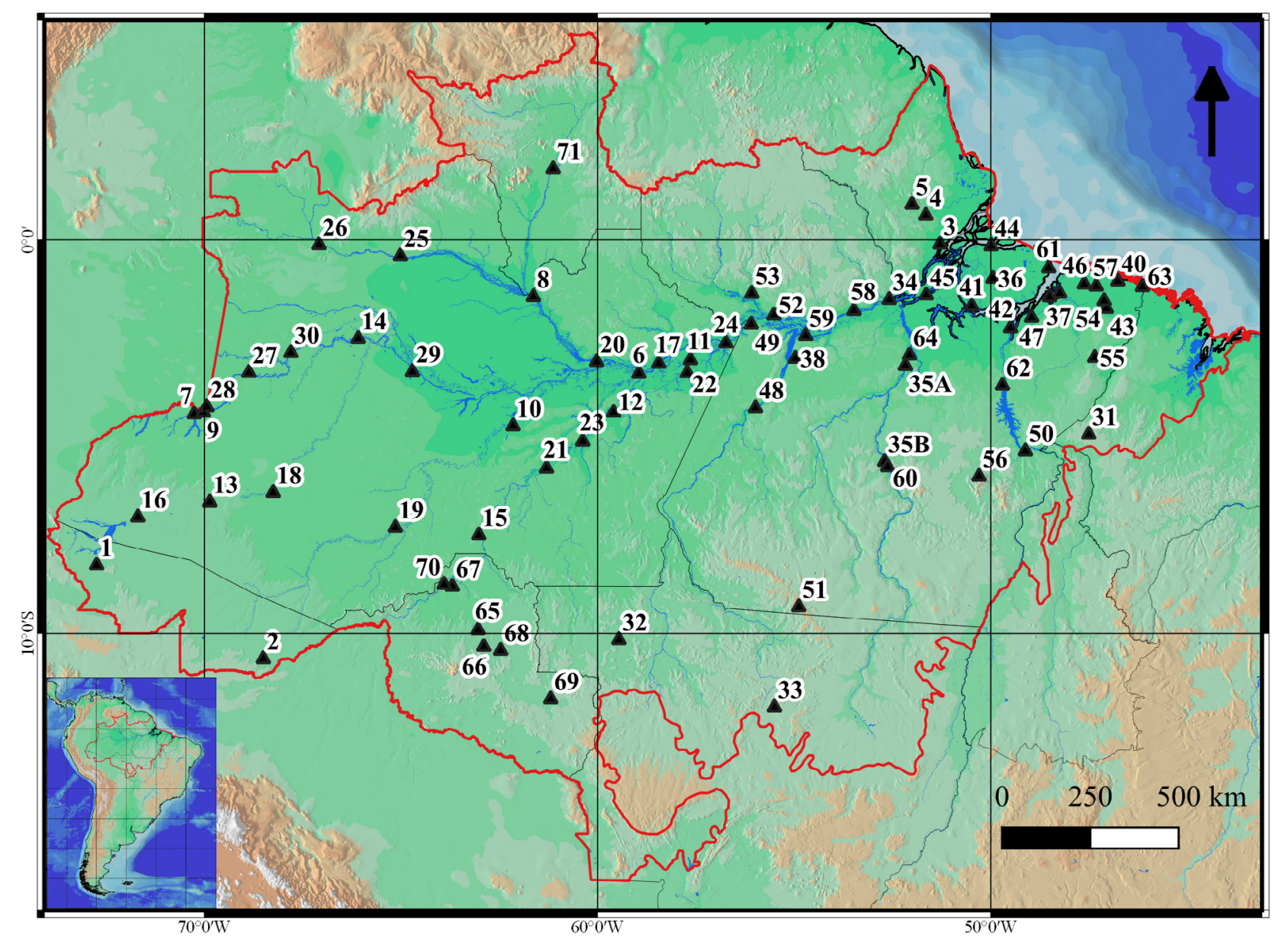

Figure 1. Geographic distribution of Euchromiina species records in the Brazillian Amazon. The numbers refer to the municipalities of Table 2. 
Table 2. Geographic coordinates of municipalities and richness of the Euchromiina species (Erebidae, Arctiinae, Arctini) in the Brazillian Amazon Biome.

\begin{tabular}{|c|c|c|c|c|c|}
\hline $\mathbf{N}^{\mathbf{0}}$ & State & Municipality & Richness & Latitude & Longitude \\
\hline 1 & $\mathrm{AC}$ & Porto Walter & 1 & $08^{\circ} 16^{\prime} 07^{\prime \prime} \mathrm{S}$ & $72^{\circ} 44 ' 37^{\prime \prime} \mathrm{W}$ \\
\hline 2 & $\mathrm{AC}$ & Xapuri & 1 & $10^{\circ} 39^{\prime} 06^{\prime \prime} \mathrm{S}$ & $68^{\circ} 30^{\prime} 15^{\prime \prime} \mathrm{W}$ \\
\hline 4 & AP & Porto Grande & 1 & $00^{\circ} 37^{\prime} 01 " \mathrm{~N}$ & $51^{\circ} 38^{\prime} 60^{\prime \prime} \mathrm{W}$ \\
\hline 5 & AP & Serra do Navio & 16 & $00^{\circ} 53^{\prime} 45^{\prime \prime} \mathrm{N}$ & $52^{\circ} 00^{\prime} 07^{\prime \prime} \mathrm{W}$ \\
\hline 8 & $\mathrm{AM}$ & Barcelos & 4 & $01^{\circ} 27^{\prime} 01^{\prime \prime S}$ & $61^{\circ} 37^{\prime} 59^{\prime \prime} \mathrm{W}$ \\
\hline 9 & $\mathrm{AM}$ & Benjamin Constant & 19 & $04^{\circ} 22^{\prime} 60^{\prime \prime} \mathrm{S}$ & $70^{\circ} 01^{\prime} 52^{\prime \prime} \mathrm{W}$ \\
\hline 10 & $\mathrm{AM}$ & Beruri & 1 & $04^{\circ} 44^{\prime} 09^{\prime \prime} \mathrm{S}$ & $62^{\circ} 09^{\prime} 01^{\prime \prime} \mathrm{W}$ \\
\hline 11 & $\mathrm{AM}$ & Boa Vista do Ramos & 3 & $03^{\circ} 03^{\prime} 19^{\prime \prime} \mathrm{S}$ & $57^{\circ} 38^{\prime} 20^{\prime \prime} \mathrm{W}$ \\
\hline 15 & $\mathrm{AM}$ & Humaitá & 4 & $07^{\circ} 30^{\prime} 23 " \mathrm{~S}$ & $63^{\circ} 01^{\prime} 14^{\prime \prime} \mathrm{W}$ \\
\hline 16 & $\mathrm{AM}$ & Ipixuna & 1 & $07^{\circ} 03^{\prime} 03^{\prime \prime} \mathrm{S}$ & $71^{\circ} 41^{\prime} 41^{\prime \prime} \mathrm{W}$ \\
\hline 17 & $\mathrm{AM}$ & Itacoatiara & 5 & $03^{\circ} 08^{\prime} 36^{\prime \prime} \mathrm{S}$ & $58^{\circ} 26^{\prime} 39^{\prime \prime} \mathrm{W}$ \\
\hline 18 & $\mathrm{AM}$ & Itamarati & 1 & $06^{\circ} 25^{\prime} 31^{\prime \prime S}$ & $68^{\circ} 15^{\prime} 12^{\prime \prime} \mathrm{W}$ \\
\hline 19 & $\mathrm{AM}$ & Lábrea & 1 & $07^{\circ} 18^{\prime} 51^{\prime \prime S}$ & $65^{\circ} 08^{\prime} 40^{\prime \prime} \mathrm{W}$ \\
\hline 20 & AM & Manaus & 28 & $03^{\circ} 06^{\prime} 07^{\prime \prime} \mathrm{S}$ & $60^{\circ} 01 ' 30^{\prime \prime} \mathrm{W}$ \\
\hline 21 & $\mathrm{AM}$ & Manicoré & 9 & $05^{\circ} 48^{\prime} 34^{\prime \prime} \mathrm{S}$ & $61^{\circ} 18^{\prime} 00^{\prime \prime} \mathrm{W}$ \\
\hline 22 & $\mathrm{AM}$ & Maués & 4 & $03^{\circ} 23^{\prime} 01^{\prime \prime S}$ & $57^{\circ} 43^{\prime} 07^{\prime \prime} \mathrm{W}$ \\
\hline 23 & $\mathrm{AM}$ & Novo Aripuanã & 1 & $05^{\circ} 07^{\prime} 15^{\prime \prime} \mathrm{S}$ & $60^{\circ} 22^{\prime} 47^{\prime \prime} \mathrm{W}$ \\
\hline 29 & $\mathrm{AM}$ & Tefé & 39 & $03^{\circ} 21^{\prime} 16^{\prime \prime} \mathrm{S}$ & $64^{\circ} 42^{\prime} 40^{\prime \prime} \mathrm{W}$ \\
\hline 30 & $\mathrm{AM}$ & Tonantins & 1 & $02^{\circ} 52^{\prime} 24^{\prime \prime} \mathrm{S}$ & $67^{\circ} 48^{\prime} 08^{\prime \prime} \mathrm{W}$ \\
\hline 31 & MA & Açailândia & 33 & $04^{\circ} 56^{\prime} 49^{\prime \prime} \mathrm{S}$ & $47^{\circ} 30^{\prime} 17^{\prime \prime} \mathrm{W}$ \\
\hline 32 & MT & Aripuanã & 6 & $10^{\circ} 10^{\prime} 01 " \mathrm{~S}$ & $59^{\circ} 27^{\prime} 33^{\prime \prime} \mathrm{W}$ \\
\hline 33 & MT & Sinop & 9 & $11^{\circ} 52^{\prime} 51 " \mathrm{~S}$ & $55^{\circ} 30^{\prime} 08^{\prime \prime} \mathrm{W}$ \\
\hline 34 & $\mathrm{PA}$ & Almeirim & 3 & $01^{\circ} 31^{\prime} 24 " \mathrm{~S}$ & $52^{\circ} 34^{\prime} 54^{\prime \prime} \mathrm{W}$ \\
\hline $35 \mathrm{~A}$ & PA & Altamira $^{\dagger}$ & 29 & $03^{\circ} 11^{\prime} 55^{\prime \prime S}$ & $52^{\circ} 10^{\prime} 15^{\prime \prime} \mathrm{W}$ \\
\hline $35 \mathrm{~B}$ & PA & Altamira $\{\text { Serra do Pardo National Park }\}^{\dagger}$ & 13 & $05^{\circ} 38^{\prime} 21 " \mathrm{~S}$ & $52^{\circ} 41^{\prime} 52^{\prime \prime} \mathrm{W}$ \\
\hline 36 & PA & Anajás & 1 & $00^{\circ} 59^{\prime} 13 " \mathrm{~S}$ & $49^{\circ} 56^{\prime} 23^{\prime \prime} \mathrm{W}$ \\
\hline 37 & $\mathrm{PA}$ & Belém & 74 & $01^{\circ} 27^{\prime} 21^{\prime \prime S}$ & $48^{\circ} 30^{\prime} 15^{\prime \prime} \mathrm{W}$ \\
\hline 38 & PA & Belterra $\{\text { National Forest of Tapajós }\}^{\dagger}$ & 28 & $03^{\circ} 01^{\prime} 05^{\prime \prime} \mathrm{S}$ & $54^{\circ} 58^{\prime} 10^{\prime \prime} \mathrm{W}$ \\
\hline 39 & PA & Benevides & 1 & $01^{\circ} 21^{\prime} 42^{\prime \prime} \mathrm{S}$ & $48^{\circ} 14^{\prime} 40^{\prime \prime} \mathrm{W}$ \\
\hline 40 & $\mathrm{PA}$ & Bragança & 2 & $01^{\circ} 03^{\prime} 13^{\prime \prime S}$ & $46^{\circ} 45^{\prime} 56^{\prime \prime} \mathrm{W}$ \\
\hline 41 & $\mathrm{PA}$ & Breves & 3 & $01^{\circ} 40^{\prime} 56^{\prime \prime} \mathrm{S}$ & $50^{\circ} 28^{\prime} 49^{\prime \prime} \mathrm{W}$ \\
\hline 42 & $\mathrm{PA}$ & Cametá & 1 & $02^{\circ} 14 ' 40^{\prime \prime} \mathrm{S}$ & $49^{\circ} 29^{\prime} 45^{\prime \prime} \mathrm{W}$ \\
\hline 43 & $\mathrm{PA}$ & Capitão Poço & 11 & $01^{\circ} 44^{\prime} 48^{\prime \prime S}$ & $47^{\circ} 03^{\prime} 33^{\prime \prime} \mathrm{W}$ \\
\hline 44 & PA & Chaves & 1 & $00^{\circ} 09^{\prime} 36^{\prime \prime S}$ & $49^{\circ} 59^{\prime} 18^{\prime \prime} \mathrm{W}$ \\
\hline
\end{tabular}


Continuation Table 2.

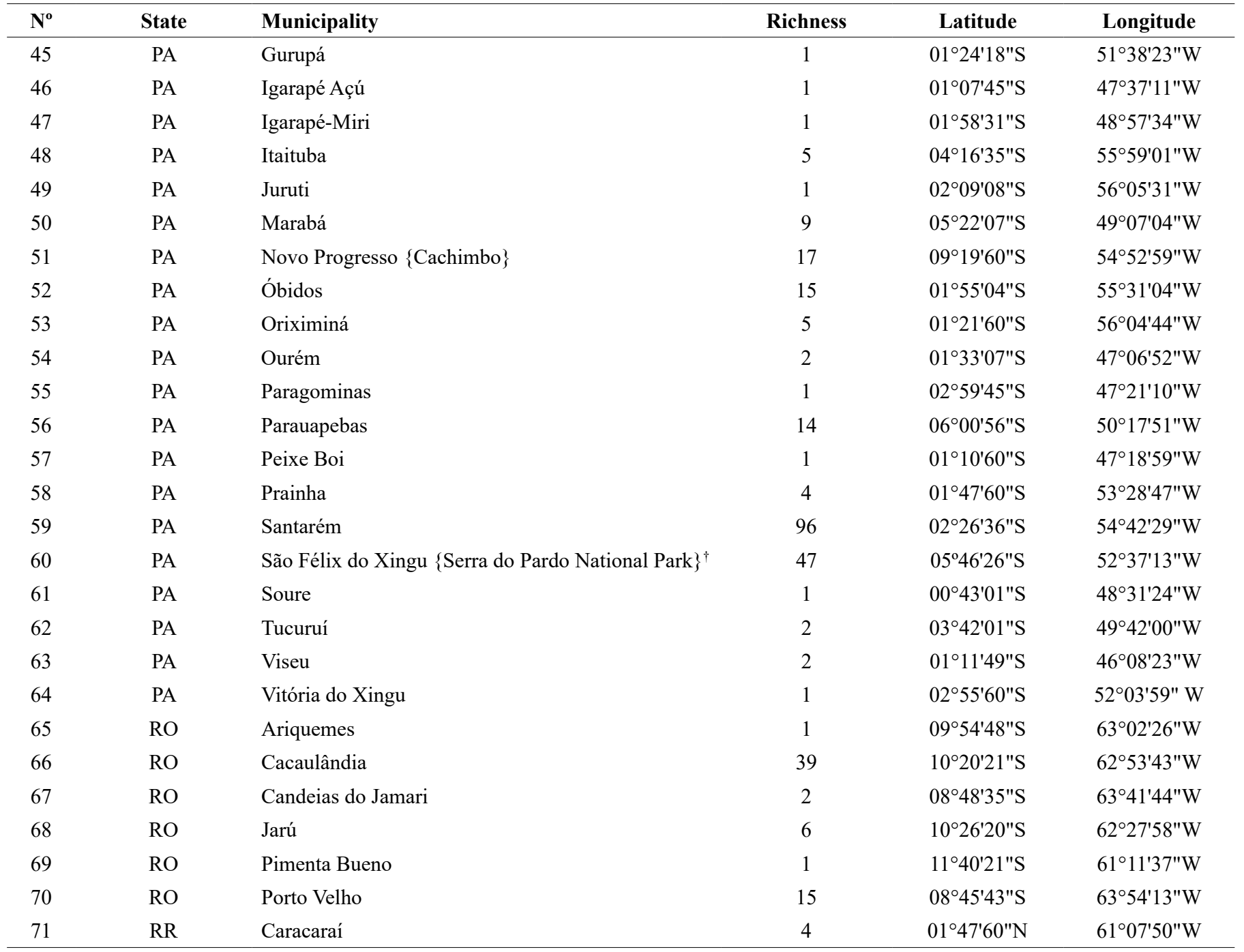

Geographic coordinates of municipality marked with ${ }^{\dagger}$ are the citations referred, other obtained by Google Earth or Geo Loc tool (see Materials and Methods).

The Euchromiina richness of Santarém and Belém were the largest recorded in Brazilian sites. For example, in Salesópolis was recorded 47 Euchromiina species (Ferro \& Diniz 2007) and in Joinville 42 species (Ferro et al. 2012). These two last sites were located in the Atlantic Forest biome and were intensively sampled. In the Cerrado sites, were recorded a much lower number of Euchromiina species, ranging from 11 (Scherrer et al. 2013) to 30 (Moreno \& Ferro 2016). Moreover, the number of Euchromiina species of a single Amazon site (Santarém, 96) was higher than the entire Cerrado Euchromiina fauna (Ferro et al. 2010). It may indicate that this taxon is more diverse in rain-forest than in xeric environments. Studies with Arctiini in the Altamira (Pará Brazilian State) showed that there are 2 times more Euchromiina species in Amazonian forest sites (64, Teston \& Correa 2015) than in Cerrado sites (32, Valente et al. 2018) and 4 times more Euchromiina species in Amazonian forest sites than altered sites (pasture and orchard) (16, Delfina \& Teston 2013). Martins et al. (2017) also obtained 2.5 times more butterfly species in sites of Amazonian forest than in Cerrado sites in the Maranhão Brazilian State.
Teston et al. (2019) have reported 847 Arctiinae species in the Brazilian Amazon. Due to the new records obtained in our study (13), there was an increase in richness for the biome, generating a total of 860 tiger moth Amazon species. As observed for the subtribes Phaegopterina (Teston \& Ferro 2016a), Pericopina (Teston \& Ferro 2016b) and Ctenuchina (Teston et al. 2019), the number of Amazonian Euchromiina species is underestimated because the fauna was never sampled in the vast majority of the biome (less than $10 \%$ of the municipalities were sampled). Even the sites already sampled need to be studied in the long-term (at least 1 year of sampling) because it is known that tiger moths respond to climate/seasonal changes (Kitching et al. 2000, Hilt et al. 2007, Scherrer et al. 2013, Ferro et al. 2014) and that Lepidoptera richness is higher in long-term surveys than in short-term surveys (Ferro \& Diniz 2007, Moreno \& Ferro 2016, Martins et al. 2017). Moreover, studies that sample Amazonian Lepidoptera in different types and strata of vegetation are rare. However, it is known that tiger moths respond to vegetation changes (Kitching et al. 2000, Ferro \& Diniz 2007, Ferro $\&$ Romanowski 2012) and some genera of Euchromiina (Macrocneme 
and Poliopastea) are canopy flyers (Brehm 2009). In order to suggest efficient conservation policies for the Amazon Arctiinae fauna, it is urgent to intensify the sampling effort in this biome, both spatially and temporally. Finally, in addition to reduce species distribution gaps, it is also necessary to invest in studies on the taxonomy of Euchromiina in order to decrease the bias of the Linnean shortfall (Whittaker et al. 2005) on diversity patterns since the taxonomy of this taxon is still complicated: there are many descriptions based on only one individual, many species to be described, and many synonyms to elucidate.

\section{Acknowledgements}

We are indebted to the museum curators for allowing VGF's access to the collections under their care and for logistical support. This publication is part of the RedeLep "Rede Nacional de Pesquisa e Conservação de Lepidópteros”, SISBIOTA-Brasil, CNPq (563332/2010-7)

\section{Author Contributions}

José Augusto Teston: Substantial contribution in the concept and design of the study.

Viviane Gianluppi Ferro; Substantial contribution in the concept and design of the study.

\section{Conflicts of interest}

The authors declare that they have no conflict of interest related to the publication of this manuscript.

\section{Data availability}

The data are deposited in the respective collections mentioned in the Material and Methods.

\section{References}

ARAGÃO, L.E.O.C., POULTER, B., BARLOW, J.B., ANDERSON, L.O., MALHI, Y., SAATCHI, S., PHILLIPS, O.L. \& GLOOR, E. 2014. Environmental change and the carbon balance of Amazonian forests. Biol. Rev. 89: 913-931.

BREHM, G. 2009. Patterns of arctiid diversity. In Tiger moths and wooly bears. Behavior, ecology, and evolution of the Arctiidae (W.E. Conner, ed.). Oxford University Press, New York, p. 223-232.

BRYK, F. 1953. Lepidoptera aus dem amazonasgebiete und aus Peru gesammelt von Dr. Douglas Melin und Dr. Abraham Roman. Ark. zool. 5(1): 1-268.

BUTLER, A.G. 1876. Notes on the Lepidoptera of the family Zygaenidae, whit descriptions of new genera and species. J. Linn. Soc. Lond., Zool. 12(60-62): 342-407.

BUTLER, A.G. 1877. Illustrations of typical specimens of Lepidoptera Heterocera in the British Museum part I. Taylor \& Francis, London.

BUTLER, A.G. 1878. On the Lepidoptera of the Amazons, collected by Dr James W. H. Trail during the years 1873 to 1875 . Trans. Entomol. Soc. Lond. 26(1): 39-84.

CASAGRANDE M.M., MIELKE O.H.H. CARNEIRO E., RAFAEL, J.A. \& HUTCHINGS, R.W. 2012. Hesperioidea e Papilionoidea (Lepidoptera) coligidos em expedição aos Rios Nhamundá e Abacaxis, Amazonas, Brasil: novos subsídios para o conhecimento da biodiversidade da Amazônia Brasileira. Rev. Bras. Entomol. 56(1): 23-28.
CONNER, W.E. \& JORDAN, A.T. 2009. From armaments to ornaments: the relationship between chemical defense and sex in tiger moths. In Tiger moths and wooly bears. Behavior, ecology, and evolution of the Arctiidae (W.E. Conner, ed.). Oxford University Press, New York, p.155-172.

DELFINA, M.C. \& TESTON, J.A. 2013. Arctiinae (Lepidoptera, Arctiidae) ocorrentes em uma área de pastagem na Amazônia Oriental em Altamira, Pará, Brasil. Acta Amaz. 43(1): 81-90.

DOGNIN, P. 1923. Hétérocères nouveaux de l'Amérique du Sud Fascicule XXI. Imprimerie Oberthür, Rennes.

DRAUDT, M. 1914-1919. Familie: Syntomidae. In Die Gross-Schmetterlinge der Erde. II. Abteilung: Die Gross-Schmetterlinge des Amerikanischen Faunengebietes. 6. Band. Die Amerikanischen Spinner und Schwärmer (A. Seitz, ed.). Alfred Kernen, Stuttgart, p.33-230.

DRAUDT, M. 1931. Neue Amatiden des amerikanischen Faunengebietes. Ent. Rdsch. 48: 45-48.

DRUCE, H. 1897. Descriptions of some new Species of Heterocera from Tropical America. Ann. Mag. nat. Hist. (sixth series) 20(117): 299-305.

DRUCE, H. 1898a. Descriptions of some new species of Heterocera. Ann. Mag. nat. Hist. (seventh series) 1(3): 207-215.

DRUCE, H. 1898b. Descriptions of some new species of Syntomidae, chiefly in the Oxford Museum. Ann. Mag. nat. Hist. (seventh series) 1(5): 401-408.

FELDER, C. \& FELDER, R. 1864-1875. Pl 75-120 (1874). In Reise der österreichischen fregatte novara um die Erde in den Jahren 1857, 1858, 1859 unter den Befehlen des Commodore B. Von Wüllestorf-Urbair. Zoologischer Theil, Zweiter Band, Zweite Abtheilung: Lepidoptera (C. Felder, R. Felder \& A.F. Rogenhofer, eds.). Staatsdruckerei, Vienna.

FERRO, V.G. \& DINIZ, I.R. 2007. Arctiidae (Insecta: Lepidoptera) da Estação Biológica de Boracéia (Salesópolis, São Paulo, Brasil). Biota Neotrop. 7(3): 331-338. http://dx.doi.org/10.1590/S1676-06032007000300033 (last access on 30/01/2019).

FERRO, V.G. \& DINIZ, I.R. 2010. Riqueza e composição das mariposas Arctiidae (Lepidoptera) no Cerrado. In Cerrado: conhecimento quantitativo como subsídio para as ações de conservação (I.R. Diniz, J. MarinhoFilho, R.B. Machado \& R. Cavalcanti, eds.). Editora Thesaurus, Brasília, p.255-313.

FERRO V.G. \& TESTON, J.A. 2009. Composição de espécies de Arctiidae (Lepidoptera) no sul do Brasil: relação entre tipos de vegetação e entre a configuração espacial do hábitat. Rev. Bras. Entomol. 53(2): 278-286.

FERRO, V.G., MELO, A.S. \& DINIZ, I.R. 2010. Richness of tiger moths (Lepidoptera: Arctiidae) in the Brazilian Cerrado: how much do we know? Zoologia 27(5): 725-731.

FERRO, V.G. \& ROMANOWSKI, H.P. 2012. Diversity and composition of tiger moths (Lepidoptera: Arctiidae) in an area of Atlantic Forest in southern Brazil: is the fauna more diverse in the grassland or in the forest? Zoologia 29(1): 7-18.

FERRO, V.G., RESENDE, I.M.H. \& DUARTE, M. 2012. Arctiinae (Lepidoptera: Erebidae) do estado de Santa Catarina, Brasil. Biota Neotrop. 12(4): 166-180. http://dx.doi.org/10.1590/S1676-06032012000400018 (last access on $30 / 01 / 2019$ ).

FERRO, V.G., LEMES, P., MELO, A.S. \& LOYOLA, R. 2014. The Reduced Effectiveness of Protected Areas under Climate Change Threatens Atlantic Forest Tiger Moths. PLoS ONE 9(9): e107792.

FREITAS, M.P. de. 2014. Estratificação vertical de Arctiini (Lepidoptera, Erebidae, Arctiinae) na Floresta Nacional do Tapajós, Amazônia Oriental, Pará, Brasil. Dissertação de Mestrado, Universidade Federal do Oeste do Pará, Santarém.

GAEDE, M. 1926. Amatiden des Berliner Zoologischen Museums (Lep.). Dtsch. entomol. z. 1926(2): 113-136.

HAGMANN, G. 1938. Syntomideos (Amatideos=Euchromideos) do Estado do Pará. In Livro Jubilar do Professor Lauro Travassos. Typographia do Instituto Oswaldo Cruz, Rio de Janeiro, p.185-194.

HAMPSON, G.F. 1898. Catalogue of the Lepidoptera Phalaenae in the British Museum. Catalogue of the Syntomidae in the collection of the British Museum. Taylor \& Francis, London. 
HAMPSON, G.F. 1901. Catalogue of the Lepidoptera Phalaenae in the British Museum. Catalogue of the Arctiadae (Arctianae) and Agaristidae in the collection of the British Museum. Taylor \& Francis, London.

HAMPSON, G.F. 1909. Descriptions of new genera and species of Syntomidae, Arctiadae, Agaristidae and Noctuiidae. Ann. Mag. nat. Hist. (eighth series) 4(22): 344-388.

HAMPSON, G.F. 1914. Catalogue of the Lepidoptera Phalaenae in the British Museum. Supplement. Volume I. Catalogue of the Amatidae and Arctiadae (Nolinae and Lithosianae) in the collection of the British Museum. Taylor \& Francis, London.

HAMPSON, G.F. 1920. Catalogue of the Lepidoptera Phalaenae in the British Museum. Supplement. Volume II. Catalogue of the Lithosiadae (Arctianae) and Phalaenoididae in the collection of the British Museum. Taylor \& Francis, London.

HAWES, J., MOTTA, C. da S., OVERAL, W.L., BARLOW, J., GARDNER, T.A. \& PERES, C.A. 2009. Diversity and composition of Amazonian moths in primary, secondary and plantation forest. J. Trop. Ecol. 25(3): 281-300.

HEPPNER, J.B. 1991. Faunal regions and the diversity of Lepidoptera. Trop. Lep. 2(suppl. 1): 1-85.

HERNÁNDEZ-BAZ, F., MORON, M.A., CHAMÉ-VAZQUEZ, E. \& GONZÁLEZ, J.M. 2013. Ctenuchina and Euchromiina (Lepidoptera: Erebidae: Arctiinae) of Three Biological Research Stations of México's Ecosur. J. Lepid. Soc. 67(3): 145-155.

HERRICH-SCHÄFFER, G.A.W. [1854]. Sammlung neuer oder bekannter ausseuropaischer Schmetterlinge 1. G.J. Manz, Regensburg.

HILT, N., BREHM G. \& FIEDLER, K. 2007. Temporal Dynamics of Rich Moth Ensembles in the Montane Forest Zone in Southern Ecuador. Biotropica 39(1): 94-104.

INPE. 2015. Amazon Program - Monitoring the Brazilian Amazon by satellite: The PRODES, DETER, DEGRAD and TerraClass Systems. Available at www.inpe.br (last access on 30/01/2019).

KAYE, W.J. 1918. Descriptions from the Joicey Collection of new Species of Syntomidae, Nymphalidae, and Hesperidae, and Two Genera of Syntomidae. Ann. Mag. nat. Hist. (ninth series) 2(9): 225-232.

KITCHING, R.L., ORR, A.G., THALIB L., MITCHELL H., HOPKINS, M.S \& GRAHAM, A.W. 2000. Moth assemblages as indicators of environmental quality in remnants of upland Australian rain forest. J. Appl. Ecol. 37: 284-297.

MACHADO FILHO, J.P. \& RÊGO BARROS, A.R. do. 1969. Estudo dos Ctenuchidae Neotropicais - III. Novo gênero para Histioea tina Walker, 1854 (Lepidoptera - Heterocera). Bol. Mus. Nac. 270(1): 1-9.

MACHADO FILHO, J.P. \& RÊGO BARROS, A.R. do. 1971. Ctenuchidae Neotropicais VI. Sobre o gênero Histioea Walker, 1854 e descrição de nova espécie (Lepidoptera - Heterocera). Bol. Mus. Nac. 284(1): 1-17.

MACHADO FILHO, J.P. \& RÊGO BARROS, A.R. do. 1973. Ctenuchidae Neotropicais VII. Sobre o gênero Histioea Walker, 1854 (Lepidoptera Heterocera). Publ. avulsas - Mus. Para. Emilio Goeldi 20(1): 247-271.

MARTINS, L.P., ARAUJO JUNIOR, E.C., MARTINS, A.R.P., COLINS, M.S., ALMEIDA, G.C.F. \& AZEVEDO, G.G. 2017. Butterfies of Amazon and Cerrado remnants of Maranhão, Northeast Brazil. Biota Neotrop. 17(3): e20170335. http://dx.doi.org/10.1590/1676-0611-bn-2017-0335 (last access on 30/01/2019).

MORENO, C. \& FERRO, V.G. 2016. Arctiinae moths (Lepidoptera, Erebidae) of the Emas National Park, Goiás, Brazil. Biota Neotrop. 16(2): e20150037. http://dx.doi.org/10.1590/1676-0611-BN-2015-0037 (last access on 30/01/2019).

MYERS, N., MITTERMEIER, R.A., MITTERMEIER, C.G., FONSECA, G.A.B. \& KENT, J. 2000. Biodiversity hotspots for conservation priorities. Nature 403(6772): 853-858.

OBERTHÜR, C. 1912. Etudes de lépidoptérologie comparée. Fascicule VI. Imprimier Oberthür, Rennes.

PEREIRA, O. de A. 1958. Contribuição ao conhecimento dos Ctenuchindae (Lep.) VIII - Gênero Orcynia Walker, 1854. Pap. Avulsos Zool. 13(14): 161-177.
PERTY, M. 1834. Delectus animalium articulatorum: quae in itinere per Brasiliam, annis MDCCXVII-MDCCCXX jussu et auspiciis Maximiliani Josephi I. Bavariae Regis Augustissimi. Impensis Editoris, Monachii.

PINHEIRO, L.R. \& DUARTE, M. 2013. Taxonomic notes on Ctenuchina, Euchromiina, and Phaegopterina (Lepidoptera, Erebidae, Arctiinae, Arctiini). Fla. Entomol. 96(2): 351-359.

PINHEIRO L.R, \& GAAL-HASZLER, S. 2015. Illustrated catalogue of Neotropical Ctenuchina, Euchromiina and Pericopina types (Lepidoptera, Erebidae, Arctiinae, Arctiini) described by Hans Zerny, with discussion on their taxonomic status. Zootaxa 3925(4): 505-535.

PINHEIRO, L.R. 2016. Re-evaluation of the identities of Eucereon punctatum (Guérin-Méneville, [1844]) and E. archias (Stoll, 1790), with a discussion on E. mitigatum Walker, 1857, rev. stat. (Lepidoptera, Erebidae, Arctiinae, Arctiini, Ctenuchina). Zoosystema 38(1): 127-140.

ROTHSCHILD, L.W. 1910. Descriptions of new species of Arctiinae in the Tring Museum. Novit. Zool. 17(1): 172-188.

ROTHSCHILD, L.W. 1911. New Syntomidae in the Tring Museum. Novit. Zool. 18(1): 24-45.

ROTHSCHILD, L.W. 1912. New Syntomidae. Novit. Zool. 19(1): 151-186.

ROTHSCHILD, L.W. 1931. Notes on Syntomidae (Amatidae) with descriptions of new species. Novit. Zool. 37(1): 149-158.

SANDERFORD, M.V., CORO, F. \& CONNER, W.E. 1998. Courtship behavior in Empyreuma affinis Roths. (Lepidoptera, Arctiidae, Ctenuchinae): acoustic signals and tympanic organ response. Naturwissenschaften 85: 82-87.

SANTOS, E.C., MIELKE, O.H.H. \& CASAGRANDE, M.M. 2008. Inventários de borboletas no Brasil: estado da arte e modelo de áreas prioritárias para pesquisa com vistas à conservação. Nat. Conserv. 6: 68-90.

SCHAUS, W. 1924. New species of moths in the United States National Museum. Proc. U.S. nat. Mus. 65 (2520): 1-74.

SCHERRER, S., FERRO, V.G., RAMOS, M.N. \& DINIZ, I.R. 2013. Species composition and temporal activity of Arctiinae (Lepidoptera: Erebidae) in two cerrado vegetation types. Zoologia 30(2): 200-210.

SEITZ, A. 1919-1925. 4. Familie: Arctiidae, Bärenspinner. In Die GrossSchmetterlinge der Erde. II. Abteilung: Die Gross-Schmetterlinge des Amerikanischen Faunengebietes. 6. Band. Die Amerikanischen Spinner und Schwärmer (A. Seitz, ed.). Alfred Kernen, Stuttgart, p.231-497.

SIMMONS, R.B. 2009. Adaptative coloration and mimicry. In Tiger moths and wooly bears. Behavior, ecology, and evolution of the Arctiidae (W.E. Conner, ed.). Oxford University Press, New York, p. 115-126.

TESTON, J.A., ABREU, D.S. de \& FERRO, V.G. 2019. Arctiini Leach, [1815] (Lepidoptera, Erebidae, Arctiinae) of the Brazilian Amazon. III - Subtribe Ctenuchina Kirby, 1837. Biota Neotrop. 19(2): e20180673. http://dx.doi. org/10.1590/1676-0611-bn-2018-0673 (last access on 10/05/2019).

TESTON, J.A. \& CORREA, D. do C.V. 2015. The Arctiini (Lepidoptera, Erebidae, Arctiinae) fauna of the Serra do Pardo National Park, Pará, Brazil. Check List 11(2): 1-9.

TESTON, J.A. \& DELFINA, M.C. 2010. Diversidade de Arctiinae (Lepidoptera, Arctiidae) em área alterada em Altamira, Amazônia Oriental, Pará, Brasil. Acta Amaz. 40(2): 387-396.

TESTON, J.A. \& FERRO, V.G. 2016a. Arctiini Leach, [1815] (Lepidoptera, Erebidae, Arctiinae) of the Brazilian Amazon. I - Subtribe Phaegopterina Kirby, 1892. Check List 12(2): 1-16.

TESTON, J.A. \& FERRO, V.G. 2016b. Arctiini Leach, [1815] (Lepidoptera, Erebidae, Arctiinae) of the Brazilian Amazon. II - Subtribe Pericopina Walker, [1865]. Check List 12(6): 1-7.

TESTON, J.A., NOVAES, J.B. \& ALMEIDA JUNIOR, J.O.B. 2012. Abundância, Composição e Diversidade de Arctinae (Lepidoptera, Arctiidae) em um fragmento de floresta na Amazônia Oriental em Altamira, PA, Brasil. Acta Amaz. 42(1): 105-114.

TRAVASSOS FILHO, L. 1940. Contribuição à Zoogeografia dos Euchromiidae Brasileiros. I. Material colhido em Ilha Sêca, Estado de São Paulo e Salobra, Estado de Mato Grosso, de fevereiro a março de 1940. Arq. Zool. Est. São Paulo. 2(10): 281-297. 
VALENTE, D.M.P., ZENKER, M.M. \& TESTON, J.A. 2018. Tiger-Moths in Savannas in Eastern Amazon: First Assessment of Diversity and Seasonal Aspects. Neotrop. Entomol. 47(6): 842-851.

VIEIRA, I.C.G., TOLEDO, P.M., SILVA J.M.C. \& HIGUCHI, H. 2008. Deforestation and threats to the biodiversity of Amazonia. Braz. J. Biol. 68(4, Suppl.): 949-956.

VINCENT, B. \& LAGUERRE, M. 2014. Catalogue of the Neotropical Arctiini Leach, [1815] (except Ctenuchina Kirby, 1837 and Euchromiina Butler, 1876) (Insecta, Lepidoptera, Erebidae, Arctiinae). Zoosystema 36(2): 137-533.

WALKER, F. 1854a. List of the specimens of lepidopterous insects in the collection of the British Museum. Part I. - Lepidoptera Heterocera. Edward Newman, London.

WALKER, F. 1854b. List of the specimens of lepidopterous insects in the collection of the British Museum. Part II. - Lepidoptera Heterocera. Edward Newman, London.

WALKER, F. 1856. List of the specimens of lepidopterous insects in the collection of the British Museum. Part VII. - Lepidoptera Heterocera. Edward Newman, London.

WALKER, F. [1865]. List of the specimens of lepidopterous insects in the collection of the British Museum. Part XXXI. - Supplement. Edward Newman, London.

WATSON, A., FLETCHER, D.S. \& NYE, I.W.B. 1995. Noctuoidea: Arctiidae, Cocytiidae, Ctenuchidae, dilobidae, Dioptidae, lymantriidae, Notodontidae, Strepsimanidae, Thaumetopoeidae \& Thyretidae. In the Generic names of moths of the world (I.W.B. Nye, ed.). The Natural Histori Museum, London, v.2, p.1-228.
WELLER, S.J., DACOSTA, M., SIMMONS, R.B., DITTMAR, K. \& WHITING, M. 2009. Evolution and taxonomic confusion in Arctiidae. In Tiger Moths and Wolly Bears, Behavior, Ecology and Evolution of the Arctiidae (W.E. Conner, ed.). Oxford University Press, New York, p.11-30.

WELLER, S.J., SIMMONS, R.B., BOADA, R. \& CONNER, W.E. 2000 Abdominal Modifications Occurring in Wasp Mimics of the CtenuchineEuchromiine Clade (Lepidoptera: Arctiidae). Ann. Entomol. Soc. Am. 93(4): 920-928.

WHITTAKER, R.J., ARAÚJO, M.B., PAUL, J., LADLE, R.J., WATSON, J.E.M. \& WILLIS, K.J. 2005. Conservation biogeography: assessment and prospect. Divers. Distrib.:11: 3-23.

ZAHIRI, R., HOLLOWAY, J.D., KITCHING, I.J., LAFONTAINE, J.D., MUTANEN, M. \& WAHLBERG, N. 2012. Molecular phylogenetics of Erebidae (Lepidoptera, Noctuiodea). Syst. Entomol. 37(1): 102-124.

ZERNY, H. 1912. Syntomidae. In Lepidopterorum Catalogus (H. Wagner, ed.). W. Junk, Berlin, p.1-179.

ZERNY, H. 1931. Ergebnisse einer zoologischen Sammerlreise nach Brasilien, insbesondere in das Amazonasgebiet, ausgeführt von Dr. H. Zerny. VII. Teil. Lepidoptera III: Die Syntomiden des Staates Pará. Ann. Nat. hist. Mus. Wien 45(1): 225-263.

YACK, J.E. 2004. The structure and function of auditory chordotonal organs in insects. Microscop. Res. Tech. 63: 315-337. 